\title{
EL VIDEOJUEGO “EL MISTERIO DE LA PIRÁMIDE”, UNA PROPUESTA INTERACTIVA PARA EL APRENDIZAJE DE LAS MATEMÁTICAS
}

\section{THE VIDEO GAME "EL MISTERIO DE LA PYRAMIDED” (THE MYSTERY OF THE PYRAMID), AN INTERACTIVE PROPOSAL FOR THE LEARNING OF MATHEMATICS}

\author{
Guillermo Carvajal Gutiérrez \\ Institución Educativa Carlota Sánchez \\ guillermo.carvajal@ucp.edu.co \\ Paola Andrea Rojas Zambrano \\ Inst. Educativa Carlos Eduardo Vasco \\ paola.rojas@ucp.edu.co
}

\author{
Euclides Murcia Londoño \\ Universidad Católica de Pereira \\ Euclides.murcia@ucp.edu.co
}

Resumen: Este artículo muestra la implementación del videojuego "El Misterio de la Pirámide" producto del proyecto "EI videojuego como agente motivador en el aprendizaje de las matemáticas" realizado en la Especialización en Edumática de la Universidad Católica de Pereira. El juego se implementó con estudiantes de la Escuela Esperanza Planes de Pereira y tuvo como objetivo principal fortalecer el pensamiento geométrico y espacial, específicamente resolver problemas reales traduciéndolos al lenguaje geométrico en el plano y en el espacio. La metodología en que se enmarcó esta investigación buscó medir el impacto del videojuego en las competencias geométricas, la motivación y las habilidades lingüísticas; para esto se realizó un diseño de investigación de carácter Mixto, abordando lo cuantitativo desde la geometría y lo cualitativo desde la motivación y las habilidades lingüísticas. Luego de haber implementado el videojuego, como parte fundamental de esta investigación se concluye que este tipo de desarrollos tecnológicos convierten las matemáticas en algo atractivo y emocionante.

Palabras Claves: Videojuego, Motivación, Geometría, Habilidades lingüísticas, Educación, Plano, Espacio, Diseño, Tecnología, Matemáticas.
Abstract: This article shows the implementation of the video game "El Misterio de la Pirámide" product of the project "The game as a motivator in learning mathematics" developed in the Specialization in Edumática of the Catholic University of Pereira. The game was implemented with students from school Esperanza Planes in Pereira and its main objective is to strengthen the geometric and spatial thinking, specifically solve real problems translating the geometric language in the plane and in the space. The methodology in this research is framed sought to measure the impact of the game in geometric skills, motivation and language skills; for this was made a mixed research design, from addressing the quantitative with geometry and qualitative from the motivation and language skills. After implementing the game, as a fundamental part of this research concludes that such technological developments make mathematics into something attractive and exciting.

Keywords: Videogame, Motivation, geometry, language skills, Education, Plane, Space, Design, Technology, Mathematics. 


\section{Introducción}

El aprendizaje de las matemáticas ha sido de baja motivación, traumático, pesado y poco práctico, siendo conscientes de la importancia de esta ciencia y de los retos que se presentan en la actualidad para la educación, es necesario replantear la forma en que se aprenden los contenidos referentes a las temáticas correspondientes a esta disciplina, es decir una forma de lograr educar y al mismo tiempo entretener, lo que conocemos actualmente como "edutainment".

... Las matemáticas deben ser una de las áreas del conocimiento menos populares en el común de la gente. En el banco de una plaza, en el café o en el tiempo libre, es más usual ver a las personas tratando de desentrañar un tratado de filosofía, interesarse por un relato histórico o dar una mirada a las últimas noticias; que despuntar el vicio en la resolución de un problema de aritmética o de trigonometría. (IberoAmericanos, 2015)

En este sentido y teniendo en cuenta que las dificultades en el aprendizaje de las matemáticas permiten identificar diferentes factores necesarios para generar un cambio en el paradigma convencional de la enseñanza de esta ciencia. Se hace necesario

- primero realizar un cambio de los ambientes convencionales de aprendizaje que generan una baja motivación;

- segundo, establecer una la relación de los contenidos enseñados con el contexto del estudiante

- y tercero, los ambientes de aprendizaje deben estar expresados en formas y lenguajes habituales para los estudiantes.

La propuesta de este trabajo muestra una innovación en la práctica pedagógica, en la cual se abordan los tres aspectos ya mencionados, se quiere además por medio del videojuego fortalecer el pensamiento geométrico y espacial, por ser este un medio de comunicación más cercano a los estudiantes y porque siendo de alta motivación puede influenciar de una forma directa en el entorno de aprendizaje y más precisamente impactar en diferentes aspectos del estudiante, como sus habilidades lingüísticas y la relación con su entorno a través de la geometría.

Teniendo en cuenta las consideraciones anteriores este trabajo se enfocará en el fortalecimiento del pensamiento geométrico y espacial por ser este más cercano al contexto y las relaciones que el estudiante tiene con su entorno. Este tipo de pensamiento puede ser llevado a lo material, a lo tangible y el estudiante puede realizar construcciones de dichos conceptos a partir de objetos cotidianos. Por ello esta investigación se centra en determinar el impacto de la implementación del videojuego "El misterio de la pirámide" en la motivación, las habilidades lingüísticas y el pensamiento geométrico y espacial de los estudiantes de la escuela Esperanza Planes (Pereira-Risaralda).

\section{El videojuego}

... Si bien las investigaciones no son definitivas, la mayoría de ellas indican que muchos videojuegos favorecen el desarrollo de determinadas habilidades, de atención, concentración espacial, resolución de problemas, creatividad, etc. por lo que se concluye que en su conjunto, desde el punto de vista cognitivo, los VJ suponen algún tipo de ayuda en el desarrollo intelectual (Mandinacht, E. 1987; 
White, B., 1984; Okagaki, L y Frensch, P, 1994). Se sugiere que quienes juegan a los VJ(Videojuegos) adquieren mejores estrategias de conocimiento, modos de resolver problemas, se benefician en sus habilidades espaciales y aumenta su precisión y capacidad de reacción. No hay evidencia de los efectos contrarios. (Vasco)

Haciendo un poco de historia, se dice que los videojuegos tienen origen luego de la segunda guerra mundial con la aparición de las primeras computadoras, y es como a partir de ese momento se empezó a desencadenar una revolución en las formas de entretenimiento, al poco tiempo empezaron a aparecer juegos de diferente tipo como acción, aventura, deportes, retos mentales, entre otros.

En el momento en que las computadoras iniciaron la revolución tecnológica se vio la necesidad de mediar los procesos educativos por medio de dichas tecnologías, aunque algunos juegos no fueron diseñados para el aprendizaje, muchos de ellos generaron en los jugadores diferentes habilidades y aprendizajes. Como lo menciona (McFarlane, 2002: pág 219-232) los videojuegos son una herramienta que mejora las capacidades motoras y de pensamiento, además estimula la capacidad lógica.

Al respecto, se puede decir que en la educación se ha venido dando una transformación desde sus prácticas, no solo por los modelos pedagógicos liberadores, sino que también demanda una transformación en la didáctica, aunque en la virtualidad existen varios videojuegos que han sido creados con fines educativos, no siempre a través de su uso se obtienen los resultados esperados. Aunando en la situación, se ha encontrado que los videojuegos y la educación tienen un conflicto de extremos, algunos videojuegos son tan educativos que no logran satisfacer al estudiante y otros que satisfacen al estudiante no tienen según el maestro, suficiente potencial educativo. Así pues el desarrollo de videojuegos educativos debe encontrarse en un justo medio que responda a las necesidades educativas y que al mismo tiempo sea altamente motivador para el estudiante. (Padilla, 2010: pág 36)

\section{El juego en el aprendizaje}

El juego es una característica de todas las especies, si bien es practicado podemos concluir que significa una ventaja, (Gross, citado por Martínez 2008: pág 10). El juego más allá de un acto instintivo de las especies involucra un fenómeno social. (Vigotsky 1978: pág 9) dice que el juego viene de una necesidad de tener contacto con los demás, además le permite saltar de su realidad y experimentar sus deseos no logrados y a pesar de usar la imaginación le permite al niño relacionarse con la realidad.

Por su parte en la educación el juego desarrolla un papel muy importante, pues por medio de este se logra una mayor motivación en los estudiantes, podemos observar que en las escuelas a medida que el estudiante va madurando se van dejando este tipo de prácticas aun cuando son los procesos educativos lúdicos los que ellos prefieren.

\section{La geometría en la relación del hombre con su entorno}

Según los estándares establecidos por el MEN(Ministerio de Educación Nacional) el pensamiento geométrico y espacial está ubicado dentro de los cinco pensamientos en que se categoriza el pensamiento matemático. La geometría es una disciplina que 
tiene notable relevancia para el estudiante pues ella le permite ubicarse como sujeto espacial en el entorno que lo rodea.

En consecuencia el pensamiento espacial y el sistema geométrico se define como

"el conjunto de los procesos cognitivos mediante los cuales se construye y se manipula las representaciones mentales de los objetos del espacio, las relaciones entre ellas, sus transformaciones, y sus diversas traducciones o representaciones materiales" (MEN, 1998, pág 61).

Mientras tanto, en muchos campos del ser humano como la ubicación espacial, elaboración de mapas, construcción, diseño, medidas etc, se observa la importancia de esta disciplina, por tanto la geometría surge de la necesidad de relacionar al hombre con su entorno. (Gálvez, 1994: 273 -299).

\section{El videojuego como agente motivador del aprendizaje de la geometría}

Si se pensara en un ambiente escolar de excelencia para poder realizar de forma agradable el ejercicio educativo, uno de los factores más relevantes estaría relacionado con la parte volitiva del estudiante, sin lugar a duda la actitud que presenta el estudiante frente a la clase es una variable determinante para el proceso de aprendizaje, se puede decir que aún mediante una clase de baja motivación o descontextualizada, el estudiante con su disposición puede lograr aprendizajes.

En cuanto a la motivación según (Enwistle \& Waterston 1988: 258-265) está relacionada con los enfoques de aprendizaje, pues según el tipo de motivación el estudiante puede lograr un mayor tipo de comprensión. En el proceso de aprendizaje el enfoque profundo parte de la motivación intrínseca, es decir, simplemente un deseo interior por aprender, el estudiante realiza una interacción vivencial con el contenido por medio de sus conocimientos previos y su experiencia personal.

No obstante, la educación está demandando este proceso, no solo la adquisición de conceptos, sino también el desarrollo de habilidades que le permitan al estudiante desenvolverse en su ámbito socio-cultural, entre estas habilidades se destacan las lingüísticas interpretar, argumentar y proponer que según las pruebas externas (ICFES-PISA) corresponden a competencias esenciales para el desarrollo de los estudiantes.

Es así como esta investigación pretende realizar un cambio en la forma en que se enseñan las matemáticas, destacando primero la motivación para generar un cambio en la actitud del estudiante y segundo que los aprendizajes deben tener como objetivo mejorar sus habilidades comunicativas. Para abordar esta reforma se justifican los siguientes aspectos: se requiere que el maestro motive los procesos de aprendizaje haciendo que los estudiantes sean parte activa de su proceso como lo afirma (Vygostsky, 1978: 9), los estudiantes pueden ser motivados por medio del juego y con el uso de herramientas con las que los estudiantes estén familiarizados.

Sin embargo, según (Castells, 2000: pág 3) la revolución de la tecnología y la información han tenido la capacidad de penetrar todo ámbito de la de la actividad humana, teniendo en cuenta esto se requiere de una práctica educativas motivada por medio de las TIC y que permita generar en el estudiante la adquisición de competencias geométricas y espaciales. 
El videojuego "El Misterio de la Pirámide" es una herramienta TIC creada para enseñar la geometría de una forma lúdica, combinando objetos reales y virtuales en situaciones cercanas al contexto de los estudiantes. Esta aplicación reúne estrategias para motivar el aprendizaje pero también requiere de una metodología de aplicación que desarrolle al máximo habilidades de comunicación que le permita al estudiante tener un enfoque profundo de su aprendizaje, una implementación que no lo aísle si no que convoque a maestros y a estudiantes en una dinámica de aprendizaje.

\section{Implementación del videojuego (Metodología)}

Como lo indica (Plutchik, 1975: págs. 23-49) para la investigación es importante la forma en que se disponen las condiciones de un experimento pues la finalidad es llegar a un resultado óptimo. Para el adecuado diseño experimental se requiere de un método que tenga en cuenta la población, el tipo de experimento etc.

Inicialmente se tuvo en cuenta que al no seleccionar la muestra de forma aleatoria el proyecto presenta un diseño cuasi experimental, este tipo de diseño se justificó debido al bajo número de estudiantes, al usar todos los estudiantes como sujetos de estudio, se reduce el tiempo y los recursos de experimentación y además se pueden encontrar resultados de tendencias generales.

Para el diseño del presente trabajo se propuso un diseño de investigación mixta, el objetivo fue establecer el impacto de un videojuego en el aprendizaje de la geometría en los estudiantes de grado 4to y 5to de la Escuela Esperanza Planes de la Vereda Tres Puertas en el corregimiento de Morelia. La Escuela utiliza el modelo de Escuela Nueva.

Para determinar el impacto del juego en los estudiantes se estudiaron 3 variables de control, la primera variable es el rendimiento académico de los estudiantes en los desempeños alrededor de la Geometría desde 3 competencias:

- Comunicación, representación y argumentación

- Planteamiento y resolución de problemas

- Razonamiento y argumentación

La segunda variable fue las habilidades lingüísticas del estudiante desde una observación participante.

La tercera variable es la actitud del estudiante frente al estudio de la geometría, se trata con ello de verificar si le agrada o no, si le encuentra una aplicación a su cotidianidad y cómo es la motivación respecto a las clases desarrolladas en la Escuela.

La Escuela cuenta con 9 estudiantes entre 4to y 5to grado, distribuidos así: 8 de grado 4to y 1 de grado 5to; para la presente investigación se usó el diseño de grupos apareados, debido al bajo número de la población estudiada y al diseño inicial planteado. La metodología para determinar el impacto del juego se llevó a cabo de la siguiente manera:

\subsection{Prueba diagnóstica (pre-test)}

Una prueba preliminar para determinar el estado de las competencias en geometría y un video entrevista para analizar el estado de las habilidades lingüísticas y la motivación frente a las clases. 


\subsection{Intervención}

Para la intervención con el videojuego se dividieron los estudiantes en dos grupos, uno de cuatro y uno de cinco, acorde a los recursos tecnológicos disponibles.

\subsubsection{Recursos disponibles para la intervención}

5 computadores con cámara Web que contienen el videojuego y la aplicación de realidad aumentada "Aumentaty viewer".

Computador de escritorio, Video beam.

Cámara de video y fotográfica.

5 geoplanos y bandas elásticas de colores.

5 juegos de 40 dados cada uno.

5 juegos de marcas para realidad aumentada.

\subsection{Diseño Instruccional}

Para cada grupo se planearon 3 intervenciones:

a) Manejo de instrumentos (Geoplano, Dados y realidad aumentada) (1 Hora)

En esta etapa se realizaron prácticas de plano cartesiano utilizando el geoplano, se construyeron figuras tridimensionales usando dados y se utilizó la aplicación Aumentaty para realidad aumentada.

b) Utilización del videojuego (Etapa exploratoria) (2 Horas)

Se realizaron prácticas exploratorias sobre el manejo del videojuego, el personaje principal, los objetivos y el manejo de los diferentes controles.

c) Utilización del videojuego (Implementación) (3 Horas)

Se utilizó el videojuego permitiendo la interacción de los estudiantes entre sí y con los diferentes elementos que componen la ayuda didáctica. En esta etapa se realizó un video para observar las habilidades lingüísticas del estudiante y la motivación.

\subsection{Observaciones}

Las intervenciones se realizaron en jornada complementaria entre las $2 \mathrm{pm}$ y $4 \mathrm{pm}$, un horario generalmente muy difícil para estudiantes que ya han cumplido con su jornada habitual durante la mañana.

En la intervención del primer grupo uno de los estudiantes no asistió debido a que sus padres no lo autorizaron, por lo tanto se realizó la práctica con los tres estudiantes que se presentaron.

\subsection{Prueba posterior (post test)}

Teniendo en cuenta la metodología se elabora una prueba para determinar si se logró una mejoría en la adquisición del saber disciplinar, en este caso la geometría. 


\section{Resultados}

\subsection{Entrevista}

Plano cartesiano

¿Qué es el plano cartesiano? ¿Para qué se utiliza el plano cartesiano?

En esta pregunta los estudiantes no conocen una definición específica de plano cartesiano, pero algunos de ellos manifiestan uno de sus usos que es para la ubicación.

Figuras planas

¿Qué son figuras planas? ¿Para qué se utilizan las figuras planas? ¿Menciona las figuras planas que conoce?

En esta pregunta los estudiantes no saben una definición formal de figuras planas ni su uso, pero sí reconocen las figuras planas más simples (triángulos, cuadrados, entre otros).

Figuras tridimensionales

¿Qué elementos del salón son como...

Una esfera...., Un cilindro..., Un cono..., Una pirámide...

Los estudiantes reconocen las figuras tridimensionales más simples y pueden relacionarlas con su entorno. Relacionan la esfera con los balones, el cilindro con recipientes o el rollo de papel y el cono con los conos naranja usados en educación física.

Área

¿Qué es el área? ¿Para qué sirve calcular el área? ¿De qué tamaño es tu casa?

Los estudiantes no saben qué es el área ni cuál es su uso; la pregunta final tiene la intención de que el estudiante vea la necesidad de medir áreas, al preguntarle simplemente responde grande, pequeña o simplemente dicen que no saben.

\section{Sólidos}

¿Qué es el volumen? ¿Para qué se utilizan las medidas de volumen? Muestre más o menos que cantidad de agua consumen en su casa por día

Al igual que el bloque de preguntas anterior no saben qué es el volumen y para que se calcula, al preguntarle sobre el consumo de su casa se les observa una noción de volumen desde la medida de capacidad pero con recipientes comunes a sus entorno como baldes, o el tanque.

Solución de problemas de sólidos

Si llega a faltar agua en tu casa ¿Cuánta agua deben juntar para pasar el día? ¿Con cuántos baldes de agua se puede llenar el tanque de tu casa?

La intención de las preguntas está orientada a poder establecer que los estudiantes realmente no usan la precisión matemática y por tanto presentan inconsistencias, es así como siendo la pregunta (Muestre más o menos que cantidad de agua consumen 
en su casa por día) igual a la pregunta (¿Cuánta agua deben juntar para pasar el día?) solo una niña estableció que sería la misma cantidad de agua.

Para concluir podemos resaltar los siguientes aspectos: Se observa falta de motivación en el aprendizaje, los estudiantes no conservan ciertos conocimientos fundamentales de la disciplina y la mayor parte de las preguntas fueron respondidas más desde la experiencia de su cotidianidad que desde la aplicación de los conceptos geométricos en su vida diaria.

También se evidencia que la mayoría de los estudiantes responden de una manera inmediata sin realizar una debida reflexión, si bien interpretan las preguntas no buscan una solución, u otra forma de explicar el concepto, por tanto no se evidencia en ninguno de ellos una debida argumentación y mucho menos proponen diferentes soluciones a las preguntas que se le presentan.

Atendiendo a estas consideraciones, aunque se puede evidenciar según la figura $N^{\circ} 1$ que el desempeño académico de los estudiantes que participaron de la estrategia, se debe seguir motivando la enseñanza de las matemáticas en los aspectos donde los estudiantes presentan bajo nivel de desempeño.

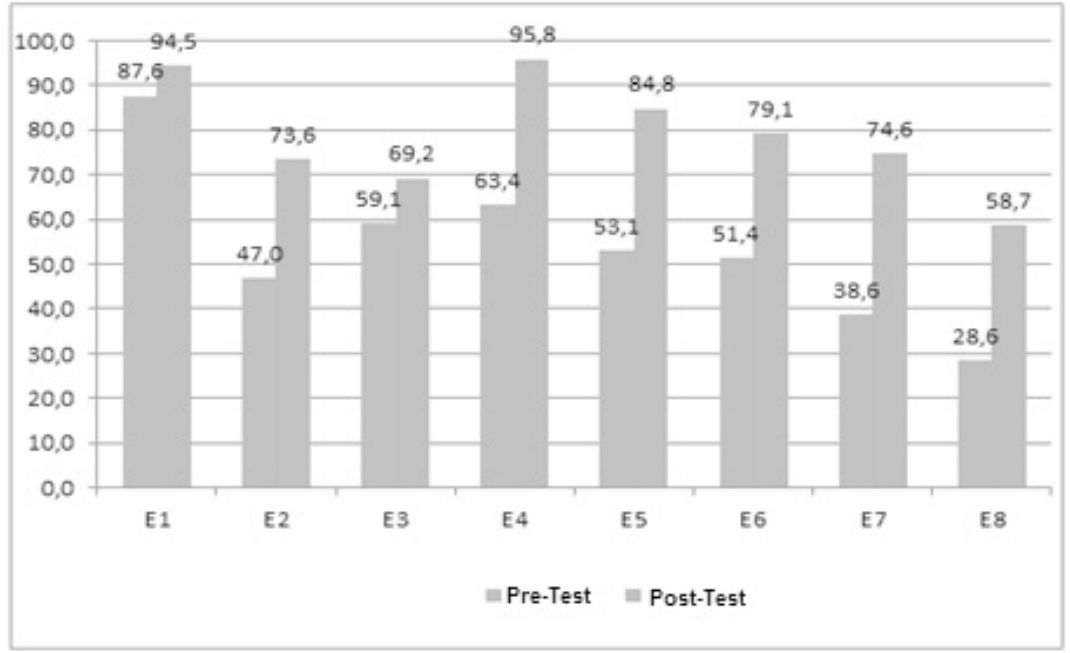

Figura 1.Comparación Pre- Test y Post-Test

Al respecto, basta precisar que en cada una de las intervenciones de esta investigación se usaron elementos o recursos que conllevaron a que los estudiantes apropiaran de manera significativa las competencias básicas en geometría propuestas en el plan de trabajo para este grupo de estudiantes.

Sobre las bases de las ideas expuestas, se muestra a continuación en la Tabla 1, como cada elementos físico aportó al desarrollo de una habilidad específica, se relaciona cada elemento con su aporte en el proceso. 
Tabla 1: Aporte al Mejoramiento de Contenidos Específicos

\begin{tabular}{|c|l|}
\hline RECURSO & \multicolumn{1}{|c|}{ APORTE AL MEJORAMIENTO DE CONTENIDOS ESPECíFICOS } \\
\hline EI Tangram & $\begin{array}{l}\text { Incluido en el juego de manera virtual permitió que los estudiantes compren- } \\
\text { dieran la noción de área o medida de superficie, además de relacionar las dife- } \\
\text { rentes figuras planas (cuadrado, rectángulos, triángulos y círculos) con objetos } \\
\text { cotidianos. }\end{array}$ \\
\hline EI Geo plano & $\begin{array}{l}\text { Incluido como elemento físico externo, aportó a los estudiantes de manera lú- } \\
\text { dica a comprender la forma en que se ubican los cuerpos en el espacio, por } \\
\text { medio de este elemento relacionaron las nociones básicas de posicionamiento } \\
\text { (derecha, izquierda, arriba y abajo). }\end{array}$ \\
\hline Los Multicubos & $\begin{array}{l}\text { Son dados que se usaron para representar la unidad cúbica como unidad bá- } \\
\text { sica de medida del volumen de cuerpos sólidos, es así como los estudiantes } \\
\text { realizaron figuras tridimensionales compuestas por unidades cúbicas. Luego } \\
\text { de usar este recurso lograron identificar elementos en tres dimensiones que } \\
\text { estaban representados de forma plana, es decir, no se limitaban a contar solo } \\
\text { las unidades visibles en la imagen sino que lograban abstraer las no visibles. }\end{array}$ \\
\hline La realidad aumentada & $\begin{array}{l}\text { Es un elemento que le permite al estudiante sumergirse en el juego y mez- } \\
\text { clar elementos reales con virtuales, por medio de esta aplicación los estudian- } \\
\text { tes identificaron de forma novedosa los elementos de los principales cuerpos } \\
\text { geométricos y los relacionaron con objetos reales de su entorno. }\end{array}$ \\
\hline
\end{tabular}

\subsection{Análisis de resultados}

Desde la perspectiva cuantitativa, con el propósito de complementar el diseño metodológico, y aunque se aclara que a pesar de no haber realizado una selección aleatoria de los estudiantes que conformaron el grupo para la experiencia, se realizó una prueba de hipótesis matemática por medio de una diferencia de medias t-student con el propósito de realizar un contraste entre los métodos usados para la enseñanza de la geometría en forma tradicional y del uso del video juego "El Misterio de la Pirámide".

\subsection{Planteamiento o formulación de la hipótesis matemática}

$\mathrm{H} 0: \mu_{2} \mu_{2} \mu_{1} \mu_{1=0}$ (hipótesis inicial o nula, la metodología tradicional entrega resultados iguales que la metodología usada mediante el videojuego "El Misterio de la Pirámide").

Ha: $\mu_{2} \mu_{2}-\mu_{1} \mu_{1>0}$ (hipótesis alternativa o del investigador, los estudiantes presentan promedio de notas mayor mediante la intervención del videojuego "El Misterio de la Pirámide").

\begin{tabular}{|c|c|c|c|c|c|c|c|c|}
\hline Estudiantes/Prueba & E1 & E2 & E3 & E4 & E5 & E6 & E7 & E8 \\
\hline Pre test & 87,6 & 47 & 59,1 & 63,4 & 53,1 & 51,4 & 38,6 & 28,6 \\
\hline Post test & 94,5 & 73,6 & 69,2 & 95,8 & 84,8 & 79,1 & 74,6 & 58,7 \\
\hline Diferencias & 6,9 & 26,6 & 10,1 & 32,4 & 31,7 & 27,7 & 36 & 30,1 \\
\hline
\end{tabular}

Tabla 2.Resultados de las pruebas y Diferencias 


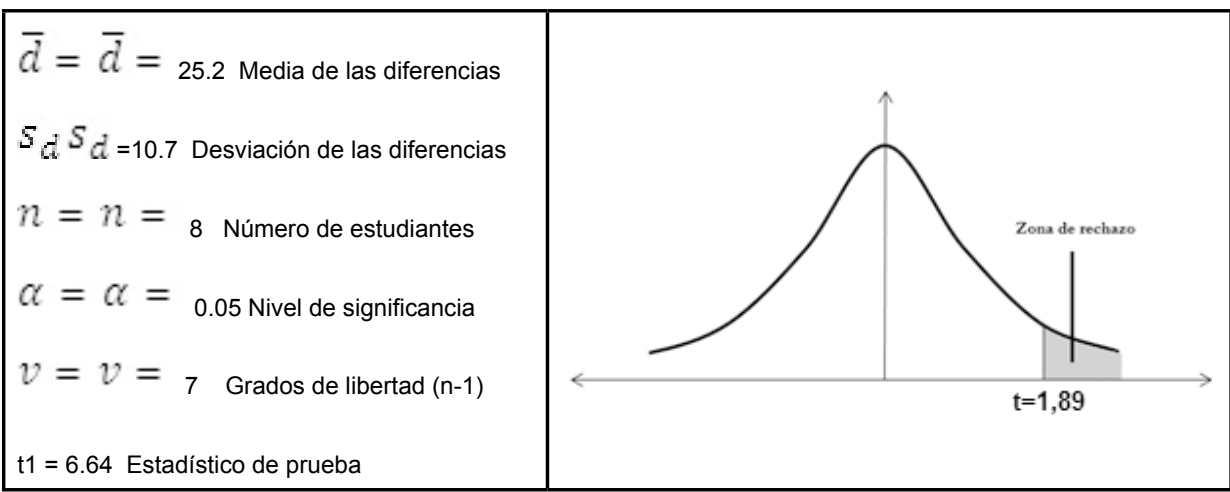

Para un nivel de significancia de 0.05 y 7 grados de libertad tenemos un estadístico de $t=1.89$. Como observamos según en el gráfico, el estadístico de prueba cae en la región de rechazo, por lo tanto se rechaza la hipótesis nula y se puede aceptar con una significancia del 5\% que la intervención del videojuego "El Misterio de la Pirámide" mejora el desempeño en geometría de los estudiantes de grado 4to y 5 to de la escuela Esperanza Planes de Pereira.

Desde la perspectiva cualitativa se realizó la transcripción y descripción de las diferentes manifestaciones de los estudiantes obtenidas en el momento de intervención, y las cuales fueron registradas mediantes videos realizados en cada una de las sesiones, con ello se realizaron las siguientes apreciaciones:

Los estudiantes ostentan que les gustó el juego, que no parece una clase de matemáticas. Seguido a ello expresan con entusiasmo y sugieren que les gustaría aprender con el juego otros temas. Los estudiantes demuestran tener habilidad en el uso de las herramientas tecnológicas, el profesor solamente realiza la guía por el proceso del videojuego.

Durante la implementación algunos estudiantes en mayor medida que otros se animan a responder las preguntas y argumentar las respuestas, acorde a esto la interacción con objetos físicos les facilita visualizar objetos tridimensionales a partir de representaciones planas.

Los estudiantes que alcanzaron mejores desempeños ayudan a los otros a realizar los objetivos, la metodología como se implementa el videojuego facilita la interacción entre el maestro, los estudiantes y la disciplina, conllevando a desarrollar un trabajo de tipo cooperativo.

\section{Conclusiones}

En la intervención se evidencia que los estudiantes además de haber enfrentado una jornada escolar completa responden a las actividades de implementación con el video juego justamente porque la dinámica del proceso educativo no es la convencional, esto refuerza que las prácticas motivadoras a través del juego mejoran la disposición y la actitud del estudiante frente a la clase.

Los test realizados demuestran que la motivación del estudiante influye significativamente en la apropiación de conocimientos, relacionados específicamente con los contenidos geométricos formales, pues en todos los estudiantes se observó así 
como se muestra en la Tabla 1, una mejoría en el manejo y apropiación de contenidos disciplinares que la educación les exige.

Por medio de un ambiente adecuado puede facilitarse el desarrollo de habilidades lingüísticas, a través del juego donde el estudiante puede manifestar este tipo de capacidades expresándose con sus compañeros y llegando a acuerdos entre sus conocimientos previos y los adquiridos.

El videojuego como las TIC son solo herramientas que facilitan la mediación en el aprendizaje, por sí solas no son suficientes, por tanto el videojuego debe estar acompañado de una adecuada metodología que potencie el fortalecimiento del pensamiento geométrico y las capacidades comunicativas.

Debido a la evolución en el tratamiento de la información de esta época y a la adquisición de nuevas competencias tecnológicas por parte de los estudiantes, los cuales han sido llamados nativos digitales, se hace necesario que los maestros implementen nuevas formas de enseñar y en general, realizar uso de la TIC, para con ello buscar motivar el aprendizaje de ciertas áreas del conocimiento que son de difícil comprensión como es el caso de las matemáticas. Es así como propuestas de las que trata este trabajo tienen un enorme potencial no solo en estas disciplinas sino en cualquier otra área de la educación.

\section{Bibliografía:}

Castells, M. (2000). La era de la información, economía sociedad y cultura, La sociedad red. Vol. I, México: Siglo XXI. Consultado 25 de Septiembre de 2014 y tomado de la URL: http://herzog.economia.unam.mx/lecturas/inae3/castellsm.pdf.

Entwistle, N.J., \& Waterston, S. (1988). "Approaches to study and levels of processing in university students", British Journal of Educational Psychology 58: (pp.258-265) http:// dx.doi.org/10.1111/j.2044-8279.1988.tb00901.x

Gálvez, G. (1994). “La geometría, la psicogénesis de las nociones espaciales y la enseñanza de la geometría en la escuela primaria”. En: Cecilia Parra e Irma Sanz (Comps) Didáctica de matemáticas. (pp.273 -299)Buenos Aires Argentina: Pairos Educador.

IberoAmericanos, O. d. (7 de Febrero de 2015). Divulgacion y Cultura Cientifica Iberoamericana. Recuperado el 15 de Octubre de 2015, de http://oei.es/divulgacioncientifica/ entrevistas_101.htm

Martínez, E. (2008). El juego como escuela de vida: Karl Groos[Revista Miscelánea de Investigación]. $n^{\circ} 22$ (pp. 7 -22) Consultada el 10 de Agosto de 20014 y tomada de URL: https://dialnet.unirioja.es/descarga/articulo/2774872.pdf

McFarlane, A., \& Sakellariou, S. (2002). The role of ICT in science education. Cambridge Journal of Education, 32, (pp.219-232). http://dx.doi.org/10.1080/03057640220147568

Ministerio de Educación Nacional (2006). Estándares básicos de Competencias en Lenguaje, Matemática, Ciencias y Ciudadanas. MEN. Bogotá.

Padilla, N. (2011) Tesis doctoral, La metodología para el diseño de videojuegos educativos. España: Universidad de Granada.

Plutchik, R. (1975). Fundamentos de investigación experimental. México: Editorial HARLA, S.A. 
Vasco, F. E.-U. (s.f.). Videojuegos y educación. Recuperado el 19 de Octubre de 2015, de http://campus.usal.es/ teoriaeducacion/rev_numero_02/n2_art_etxeberria.htm

Vygotsky, L.S. (1978). Mind in Society [La mente en sociedad]. Cambridge, Mass. Harvard University Press. 